\title{
SYSTEMIC INFLAMMATORY RESPONSE SYNDROME AND MULTIPLE ORGAN DYSFUNCTION IN PATIENTS WITH ACUTE TUMOR LYSIS SYNDROME
}

doi: 10.1590/S1807-59322009000500016

Márcio Soares, Guilherme A. Feres, Jorge I. F. Salluh

\section{INTRODUCTION}

Acute tumor lysis syndrome (ATLS) is observed in a well-defined group of patients with rapidly proliferating malignancies and results in the destruction and lysis of malignant cells. Lysis of cells results in the subsequent release of intracellular ions and metabolites into the bloodstream, thus leading to hyperkalemia, hyperphosphatemia, hyperuricemia, and hypocalcemia. This syndrome occurs either as a consequence of diverse anticancer treatments or, less frequently, spontaneously. ${ }^{1}$ Although most patients with ATLS are asymptomatic, acute renal failure (ARF), metabolic acidosis, life-threatening electrolyte abnormalities, cardiac arrhythmias, or seizures may accompany the syndrome, thereby increasing the risk of associated death. ${ }^{1,2}$

Systemic inflammatory response syndrome (SIRS) is a pathologic reaction triggered by a variety of insults, including infection, trauma, burns, and acute pancreatitis. ${ }^{3}$ SIRS becomes more severe when it is complicated by organ dysfunction. SIRS-like syndromes have been reported in patients with hematologic tumors, which reflects rapid cytoreduction. ${ }^{4}$ SIRS and multiple organ dysfunctions (MOD) associated with ATLS would seem to be physiologically plausible; however, no study has addressed this issue to the best of our knowledge. In this manuscript, we report five patients who developed ATLS associated with SIRS and MOD in the absence of clear alternative explanations.

Intensive Care Unit, Instituto Nacional de Câncer, INCA - Rio de Janeiro/ RJ, Brazil.

Emails: marciosoaresms@yahoo.com.br / marciosoaresms@gmail.com Tel.: 55212506.6120

Fax: 55212294.8620

\section{PATIENTS AND METHODS}

\section{Design and Setting}

This retrospective study was performed at a tertiary care public hospital that houses a ten-bed intensive care unit (ICU) exclusively for oncology patients, the majority of whom are referred to the center from outside hospitals. Information about the oncology ICU has been previously reported. ${ }^{5}$ The institutional review board approved this study.

From May 2000 to May 2006, the charts of every patient admitted to the ICU with a proven diagnosis of ATLS ${ }^{2}$ were reviewed. Demographic, clinical, and laboratory data were collected, including the Simplified Acute Physiology Score (SAPS) II $^{6}$ and the Sequential Organ Failure Assessment (SOFA) score. ${ }^{7}$ MOD was defined as the simultaneous presence of $\geq 2$ organ failures (SOFA score $\geq 2$ points for each system). SIRS and sepsis were diagnosed using the definitions established by the American College of Chest Physicians/Society of Critical Care Medicine consensus conferences. ${ }^{3}$ The criteria used for diagnosis of infection were those from the Centers for Disease Control. ${ }^{8}$

\section{RESULTS}

During the study period, 18 patients presenting with ATLS were identified, and MOD occurred in $11(61 \%)$ of these patients. Out of these 11 patients, 6 male patients with an average age of 52 years (range: 26-61) presented with a clinically proven infection, and five developed MOD in the absence of an additional definitive cause other than ATLS (secondary to infection). These five patients comprised the study population. Three of these patients had hematological malignancies (high-grade non-Hodgkin's lymphoma, $\mathrm{n}=1$; 
chronic lymphocytic leukemia, $\mathrm{n}=2$ ), and two patients had been diagnosed with solid tumors (germ cell tumor, $\mathrm{n}=1$; renal cancer, $\mathrm{n}=1$ ). The most frequently observed comorbidities included the following: thromboembolic disease $(n=2)$, hypertension $(n=1)$, diabetes $(n=1)$, and malnourishment $(n=1)$. Patients also presented with hyperleukocytosis $(n=1)$ or bulky/metastatic disease $(n=4)$. The primary risk factors for ATLS and significant laboratory alterations are shown in Table 1.

Table 1 - Main clinical characteristics and laboratory abnormalities $(n=5)$

\begin{tabular}{ll}
\hline Characteristics & \\
\hline Risk factors for tumor lysis syndrome & 5 \\
Large volume of tumor cells * & 3 \\
Azotemia & 1 \\
Lactate dehydrogenase $>1500 \mathrm{UI} / \mathrm{L}$ & \\
Laboratory abnormalities & 5 \\
Hyperkalemia & 4 \\
Hyperuricemia & 4 \\
Metabolic acidosis & 3 \\
Hyperphosphatemia & 2 \\
Hypocalcemia & $14.62-36.7)$ \\
C-reactive protein $(\mathrm{mg} / \mathrm{dL})$ & \\
SIRS criteria & 2 \\
Temperature criteria & 3 \\
Heart rate $>90$ bpm & 5 \\
Respiratory rate $>20$ or $\mathrm{pCO}_{2}<32 \mathrm{mmHg}$ & 5 \\
Leukocyte count criteria & 5 \\
Organ Failures & 5 \\
Respiratory & 5 \\
Renal & 5 \\
Circulatory & 5 \\
Hepatic & 5 \\
Nematological & 5 \\
\hline
\end{tabular}

* Hyperleukocytosis or bulky disease; SIRS = systemic inflammatory response syndrome

ATLS occurred spontaneously in two patients with diffuse large B-cell non-Hodgkin's lymphoma. In patients with chemotherapy-induced ATLS $(n=3)$, the treatment regimens included sunitinib for renal cancer, cisplatin and etoposide for a germ cell tumor, and cyclophosphamide and corticosteroids for chronic lymphocytic leukemia.

All patients fulfilled the criteria for SIRS on presentation to the ICU and had at least three distinct organ dysfunctions (Table 1). High levels of C-reactive protein (reference value
$<0.5 \mathrm{mg} / \mathrm{dL}$ ) were observed in all patients, and the median leukocyte count was $14.9($ range $=13.4-644.0) \times 10^{3}$ cells $/$ $\mathrm{mm}^{3}$. The average SAPS II score was 58 (range $=32-66$ ) points, and the SOFA score was 11 (range $=5-18$ ) points. All patients required ventilatory support; conventional mechanical ventilation was used in four patients, while non-invasive ventilation was used in one patient. Four patients received renal replacement therapy. Routine cultures obtained at clinical presentation were either negative $(n=4)$ or revealed non-pathogenic bacteria (Streptococcus viridans was isolated from a tracheal aspirate of one patient). Antibiotics were started empirically in three patients and were suspended after a course of three to seven days based on negative cultures results and a lack of an apparent infection site. Two patients died in the ICU as a consequence of MOD. Of the three surviving patients, two did not receive antibiotics. Full recovery of renal function was observed in two of the surviving patients.

\section{DISCUSSION}

In this study, we describe a series of patients with hematologic malignancies and solid tumors who presented with ATLS, SIRS, and MOD. Despite extensive diagnostic evaluations, neither a source of infection nor any other apparent reason for SIRS (including chemotherapeutic agents) was found in any of the patients.

Although ATLS typically occurs more frequently in patients with hematologic malignancies, it is thought that the availability of new and more effective anticancer therapies may result in an increased incidence of ATLS in patients with solid tumors. Pulmonary dysfunction and shock/hypotension have also been described in patients with hematologic tumors with intense cell lysis (especially the myelomonocytic subtype of acute myeloid leukemia). ${ }^{4} \mathrm{An}$ extensive list of clinical manifestations has been described in patients with ATLS; however, SIRS was not included in this repertoire until now. ${ }^{1,2}$

Although the etiology of SIRS in the patients described is not fully understood, it is reasonable to hypothesize that substances released by the cells during lysis may induce severe SIRS. A complex cascade of cytokines has been implicated in the development of SIRS, and this phenomenon has been well described. ${ }^{9}$ Pro-inflammatory cytokines released by cell lysis may promote nitric oxide-mediated vasodilation and alveolar inflammation, which result in hypotension and lung injury, respectively. Other substances, such as arachidonic acid metabolites and reactive oxygen species (ROS), may also induce severe inflammation. In a systemic inflammatory response, both endothelial cells and neutrophils are activated and release ROS. ${ }^{10}$ In this setting, 
ROS may perpetuate and augment the systemic inflammation and promote organ dysfunction. Moreover, serum uric acid is a potent free-radical scavenger and increases in response to oxidative stress. ${ }^{11}$ The majority (4/5) of our patients demonstrated high levels of acid uric, and only one patient had a prior history of hyperuricemia. Urate crystals may also activate proinflammatory pathways and induce a systemic inflammatory response. ${ }^{11}$ In the present study, C-reactive protein levels, a surrogate marker of systemic inflammation, were elevated in all patients.

It should be emphasized that the differentiation between non-infectious SIRS and sepsis is quite difficult. A lack of microbiologic identification in cultures is not equivalent to the absence of infection; in addition, the absence of viral infections was not systematically determined in these patients. Although two out of three of the surviving patients did not receive antibiotics, thus corroborating the association of ATLS with the development of SIRS and MOD, antibiotics should always be given urgently if the presence of infection is uncertain until it may be ruled out; this treatment strategy was the case for three of our patients.

Potential limitations of our study also include its retrospective design and small sample size. We acknowledge that the rare occurrence of these severe events lends some difficulty to studying the phenomenon in a larger population. ${ }^{4}$ Also, the lack of data on plasma levels of procalcitonin and pro-inflammatory cytokines is another potential weakness of the study. However, although the information is nonspecific, we believe that the $\mathrm{C}$-reactive protein levels provided some insight to the systemic inflammatory status of the patient because they correlate closely with circulating IL-6 levels. ${ }^{12}$

In summary, although the present study cannot establish a definite causal relationship between ATLS and SRIRS/MOD, we have demonstrated that ATLS may present in association with SIRS and MOD, the combination of which results in significant mortality. It is important that clinicians are aware of this life-threatening complication in order to effectively implement early interventions and organ support.

\section{REFERENCES}

1. Davidson MB, Thakkar S, Hix JK, Bhandarkar ND, Wong A, Schreiber MJ. Pathophysiology, clinical consequences, and treatment of tumor lysis syndrome. Am J Med. 2004;116:546-54.

2. Cairo MS, Bishop M. Tumour lysis syndrome: new therapeutic strategies and classification. Br J Haematol. 2004;127:3-11.

3. Bone RC, Balk RA, Cerra FB, Dellinger RP, Fein AM, Knaus WA, et al. Definitions for sepsis and organ failure and guidelines for the use of innovative therapies in sepsis. The ACCP/SCCM Consensus Conference Committee. American College of Chest Physicians/Society of Critical Care Medicine. Chest. 1992; 101:1644-55.

4. Hijiya N, Metzger ML, Pounds S, Schmidt JE, Razzouk BI, Rubnitz JE, et al. Severe cardiopulmonary complications consistent with systemic inflammatory response syndrome caused by leukemia cell lysis in childhood acute myelomonocytic or monocytic leukemia. Pediatr Blood Cancer. 2005;44:63-9.

5. Soares M, Salluh JI. Validation of the SAPS 3 admission prognostic model in patients with cancer in need of intensive care. Intensive Care Med. 2006;32:1839-44.

6. Le Gall J-R, Lemeshow S, Saulnier F. A new simplified acute physiology score (SAPS II) based on a European/North American multicenter study. JAMA. 1993;270:2957-63.
7. Vincent JL, Moreno R, Takala J, Willatts S, De Mendonca A, Bruining $\mathrm{H}$, et al. The SOFA (Sepsis-related Organ Failure Assessment) score to describe organ dysfunction/failure. On behalf of the Working Group on Sepsis-Related Problems of the European Society of Intensive Care Medicine. Intensive Care Med. 1996;22:707-10.

8. Garner JS, Jarvis WR, Emori TG, Horan TC, Hughes JM. CDC definitions for nosocomial infections, 1988. Am J Infect Control. 1988;16:128-40.

9. Fink MP, Evans TW. Mechanisms of organ dysfunction in critical illness: report from a Round Table Conference held in Brussels. Intensive Care Med. 2002;28:369-75.

10. Cowley HC, Bacon PJ, Goode HF, Webster NR, Jones JG, Menon DK. Plasma antioxidant potential in severe sepsis: a comparison of survivors and nonsurvivors. Crit Care Med. 1996;24:1179-83.

11. Sanchez-Lozada LG, Nakagawa T, Kang DH, Feig DI, Franco M, Johnson RJ, et al. Hormonal and cytokine effects of uric acid. Curr Opin Nephrol Hypertens. 2006;15:30-33.

12. Damas P, Ledoux D, Nys M, Vrindts Y, De Groote D, Franchimont P, et al. Cytokine serum level during severe sepsis in human IL-6 as a marker of severity. Ann Surg. 1992;215:356-62. 